\section{TBPE-Test auf basische Substanzen im Urin}

C. Vidal ${ }^{1}$ und W.-R. Külpmann ${ }^{2}$

${ }^{1}$ Landeskriminalamt Niedersachsen, Dezernat 53 „Chemie“, Hannover, Deutschland

${ }^{2}$ Hannover, Deutschland

\section{Englischer Begriff TBPE test}

Definition Nachweis basischer Substanzen (insbesondere Pharmaka) im Urin durch Reaktion mit Tetrabromphenolphthalein-Ethylester (TBPE) zu einem Farbkomplex.

Bewertung Viele basische Pharmaka, wie z. B. zentralwirksame Analgetika, Psychopharmaka, Antihistaminika, Calci- umantagonisten, $\beta$-Blocker und Muskelrelaxanzien reagieren mit TBPE unter Bildung eines Farbkomplexes. Das Verfahren ist so empfindlich, dass vielfach die Substanzen bereits in Konzentrationen $\geq 10 \mathrm{mg} / \mathrm{L}$ nachweisbar sind. Es werden je nach vorliegender(n) Substanz(en) ganz unterschiedliche Farben beobachtet: rot, grün, violett, orange. Das TBPEVerfahren kann als Screening-Verfahren im Rahmen der Vergiftungsdiagnostik eingesetzt werden.

\section{Literatur}

Lappenberg-Pelzer M (2009) Tetrabromophenolphthalein ethyl ester. In: Külpmann WR (Hrsg) Clincial toxicological analysis. Wiley-VCH, Weinheim, S 175-187 